\title{
Effects of Fluids on the Macro- and Microcirculations
}

\author{
Victoria A. Bennett", Alexander Vidouris and Maurizio Cecconi
}

\begin{abstract}
This article is one of ten reviews selected from the Annual Update in Intensive Care and Emergency Medicine 2018. Other selected articles can be found online at https://www.biomedcentral.com/collections/ annualupdate2018. Further information about the Annual Update in Intensive Care and Emergency Medicine is available from http://www.springer.com/series/8901.
\end{abstract}

\section{Background}

Intravenous fluid administration is one of the most frequently performed interventions in the intensive care unit (ICU) and in hospital in general. In fact, most inpatients will receive fluids at some point during their hospital stay [1]. In critically ill patients, fluid resuscitation is a vital component of patient management. It has been shown that both too little and too much fluid can be detrimental. A positive cumulative fluid balance on day four of a critical care admission has been associated with increased morbidity [2, 3]. Both perioperatively and during sepsis, a U-shaped curve has been described for volume of fluid administered and morbidity. Higher mortality is observed at both extremes of volume of fluid given $[4,5]$.

However, despite extensive research in the field, controversy remains regarding the best approach to fluid therapy. The FENICE study focused on the fluid challenge and found wide disparity in practice; from fluid choice to method of administration and clinician response to the result [6]. To help guide decision making around fluid administration, the effects, both desirable and potentially detrimental ones, need to be considered. This can be considered at both the macrocirculatory and the microcirculatory level. Whilst in health coherence between the macrocirculation and microcirculation can be assumed,

\footnotetext{
* Correspondence: v.bennett1@nhs.net

Department of Intensive Care Medicine, St George's University Hospital NHS Foundation Trust, London, UK
}

this is lost in some disease states. This overview explores the effects of the fluid on the macro- and microcirculations and how we can monitor these effects.

\section{Indications for Fluid}

Classically, the need for fluid therapy is identified using information from the clinical history, examination, measurement of hemodynamic variables and markers of tissue hypoperfusion [7]. Markers of hypoperfusion may include lactate, prolonged capillary refill time and skin mottling [6]. A fluid challenge is given when tissue hypoperfusion is suspected [7]. Fluid is given to optimize cardiovascular status with the aim of ensuring adequate end-organ perfusion and improving oxygen delivery to the tissues. Fluid is given as a fluid challenge so that response can be assessed and the need for ongoing fluid therapy ascertained. To mitigate against the risk of fluid overload in those who do not require additional intravascular volume, the smallest volume that provides an effective challenge of the cardiovascular system should be used [8].

Most often, measures of the macrocirculation are used to assess and treat hemodynamic compromise in the critically ill patient and measures of the microcirculation are not routinely used at the bedside. Resuscitation based on macrocirculatory endpoints is expected to result in parallel improvement in the microcirculation [9].

\section{Macrocirculation}

The macrocirculatory response to intravenous fluid administration is based on the principles of the FrankStarling law of the heart. Venous return is always equal to cardiac output. The Frank-Starling principle describes how the heart is able to accommodate increased venous return and then eject the increased volume from the heart, with an increase in stroke volume. Increased venous return increases ventricular filling, which results in increased stretch of the cardiac myocyctes. This increased stretch results in increased contractility, or in other words, the increased diastolic expansion results in 
increased systolic contraction [10,11]. Administration of fluid aims to challenge this and assess whether a patient can accommodate an increased preload with an increased stroke volume.

The hemodynamic response to a fluid challenge can be understood by considering the effects at different points on the cardiovascular system. The first change seen is an expansion of the intravascular volume. Intravascular volume can be divided into stressed and unstressed volumes. The unstressed volume fills the vessels but does not generate any pressure. The stressed volume causes stretch of the vessel walls and increases the pressure within the vessels. Mean systemic filling pressure (Pmsf) is the measurement of the pressure when there is no flow in the vessels, or in circulatory arrest. Whilst Pmsf cannot be measured under the circumstances with which it was initially described, alternative techniques have been validated [12]. If an effective fluid challenge is given, it will, at least transiently, increase the stressed volume and cause a rise in Pmsf. This increases cardiac preload, which ultimately increases cardiac output in preload-responsive patients. The response to the increase in cardiac preload can be explained by the Frank-Starling principle.

In a patient who is fluid responsive, an effective fluid challenge will result in a significant increase, of more than $10 \%$, in the stroke volume or cardiac output. If a fluid challenge is given, which is effective in significantly increasing Pmsf, but no subsequent increase in cardiac output is seen, the patient is labeled as non-responsive [13]. This is demonstrated in Fig. 1: an adequate fluid challenge administered at point $\mathrm{A}$, increases Pmsf and a significant increase in stroke volume is seen at point $B$ - this patient is fluid responsive. At $\mathrm{C}$, although an adequate fluid challenge is given, as demonstrated by an increase in Pmsf, no significant increase in stroke volume is seen at point $\mathrm{D}$ - this patient is not fluid responsive. If an inadequate fluid challenge, of too small a volume to increase Pmsf, is given at point $\mathrm{A}$, an increase in stroke volume is not seen at point $\mathrm{E}$ and the patient would incorrectly be labeled as non-responsive to fluid.

Cecconi et al. demonstrated that a change in the pressure gradient of venous return, defined as the difference between the Pmsf and central venous pressure (CVP), following a fluid challenge was seen in responders but not in non-responders. In the non-responders, the increase in Pmsf was mirrored by an increase in CVP [14]. In those that respond, the maximal change in cardiac output is seen one minute after completion of the fluid challenge. The increase in cardiac output is a transient response; a return to baseline values is seen ten minutes post-fluid administration [13].

The decision to give fluids should be based on whether an increase in cardiac output is likely to occur with fluid loading and whether it would be likely to improve tissue perfusion. These are clinical questions that the clinician should ask before considering giving fluids. A patient who is non-responsive is unlikely to benefit from further fluid loading. Not all patients who are responsive to fluid require the additional volume [8]. For example, in a study of healthy volunteers, by definition not in shock, a significant increase in stroke volume was seen following a head down tilt (mimicking a fluid challenge). Despite being fluid responsive these healthy volunteers were unlikely to need fluid resuscitation or have evidence of tissue hypoperfusion [15].

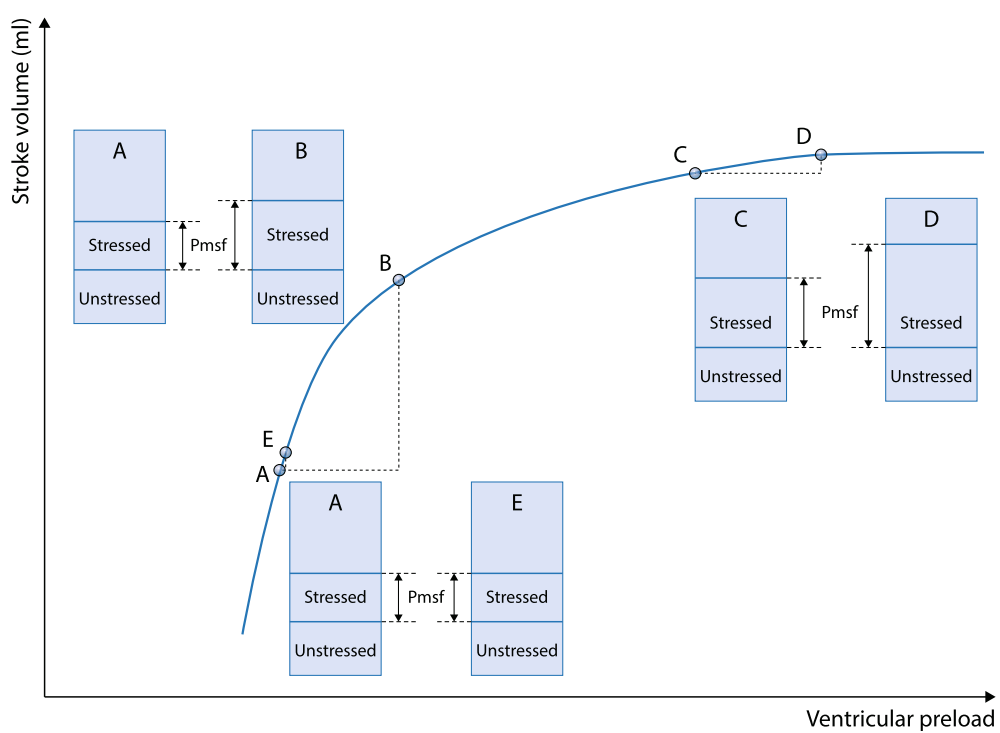

Fig. 1 Assessment of fluid responsiveness using a fluid challenge; effects on mean systemic filling pressure (Pmsf) and stroke volume, explained using the Frank Starling Curve. See text for explanation 
Other hemodynamic parameters, used more historically, include static endpoints, such as heart rate. However, a change in heart rate in response to fluid administration is not a sensitive marker of fluid responsiveness and can be influenced by numerous other factors [13].

CVP has historically also been used to guide fluid administration. Targeting a CVP of $8-12 \mathrm{cmH}_{2} \mathrm{O}$ was part of several optimization protocols in the past $[16,17]$. The role of CVP in predicting fluid responsiveness has since been refuted. Use of the CVP as an indicator of fluid responsiveness has been shown to be unreliable $[18,19]$. It does not provide accurate information about blood volume [20]. Monitoring trends in CVP over time may provide information about cardiovascular function but should not be used alone to guide fluid resuscitation [21].

\section{Microcirculation and Hemodynamic Coherence}

In health, hemodynamic coherence is assumed to exist. This means changes within the macrocirculation are reflective of changes in the microcirculation. As described earlier, the macrocirculation is generally used to guide fluid resuscitation, although ultimately the target is normalization of the microcirculation and maintenance of end-organ perfusion. However, although optimization of fluid status may result in normalization of macrocirculatory hemodynamics, such as blood pressure, this does not always translate to paralleled improvements or normalization of the microcirculation, or guarantee adequate tissue perfusion. In these conditions, a lack of hemodynamic coherence is described. This means that targeting the normalization of macrocirculatory variables may not be effective in restoring perfusion of end organs and tissues [22].

Under normal physiological conditions, the macrocirculation regulates the distribution of blood and thus end-organ perfusion. Systemic responses occur to alter macrovascular factors in order to compensate for hypovolemia, hypoxia or other nutrient delivery insufficiencies and to ensure removal of waste products. The macrocirculation is controlled by the central nervous system via the sympathoadrenomedullary axis and the parasympathetic nervous system. The renin-angiotensinaldosterone axis, vasopressin, natriuretic peptides and adipocytokines are also important in the control of blood volume and blood pressure [23]. These pathways and hormones affect the blood supply to the microcirculation via modulation of the function of the heart, the tone of the vasculature and the volume, viscosity and composition of the blood.

The microcirculation has a hugely important role in maintaining homeostasis of end organs and regulating tissue perfusion and also in thermoregulation by controlling cutaneous blood flow. Local mechanisms regulate vascular tone at the microcirculatory level by acting upon smooth muscle. They respond to physical stimuli in the microcirculation, such as increased blood pressure, causing constriction in the arterioles of the microcirculation. Some of the molecules that are active in the vasculature of the microcirculation are released from the endothelial wall including prostaglandins, nitric oxide (NO) and endothelin, which are released as a result of shear stress on the vessels. NO release can also be stimulated by other vasoactive peptides. Metabolic stimuli, such as adenosine, hydrogen ions, carbon dioxide and oxygen tension, generated in tissues also control blood flow in the microcirculation via dilation of the vessels [9]. The function of the microcirculation is also controlled by the permeability of the capillaries, their structure, the osmotic and diffusion gradients across the cell membranes and the transport systems across the vessel walls.

There may be a lack of hemodynamic coherence in disease states. States of shock, inflammation and infection can interfere with the sensing and homeostatic control mechanisms of the microcirculation [22]. Coherence is often altered in states of hemorrhagic shock or septic shock [24]. The loss of hemodynamic coherence can be the result of physiological changes in the environment resulting in nitrosative and oxidative mechanisms affecting regulation of the vasculature, changes in cell function or through changes in barrier mechanisms and concentration gradients, all of which will inhibit normal tissue perfusion. Hemodynamic coherence has been shown to vary in different tissue types dependent upon the disease state present [25].

Coherence can be lost between different tissues in a single organ system. In a pig model, resuscitation with fluids was successful in improving perfusion of the microcirculation in the mucosa of the gut, but not effective in the villi [26]. It was observed that the NO synthase (NOS) enzyme was not homogenously distributed, which caused variable and abnormal blood flow regulation in the microcirculation [27]. NOS enzymes form NO from L-arginine, which acts to decrease response to vasoactive agents. One such enzyme, NOS 3 is also utilized in the maintenance of vascular tone. $\mathrm{NO}$ is pivotal in the formation of cyclic guanosine monophosphate (cGMP), which induces smooth muscle relaxation through various mechanisms. These include activation of potassium ion channels in the cell membrane and reduction in the intracellular concentration of calcium ions. These enzymes are activated in disease states with high cytokine and endotoxin release, such as sepsis. This results in increased NO production, and subsequent dilation of the vasculature and both macrocirculatory and microcirculatory dysfunction, to varying degrees in different tissues [28]. Inappropriate vasodilation, vasoconstriction or microcirculatory tamponade induced by increased venous pressure can result in decreased oxygenation of the tissues. 
In sepsis, neutrophil adhesion and a hypercoagulable state may lead to capillary occlusion, alongside other capillaries with normal blood flow. This results in heterogeneous blood flow through the microcirculation, with subsequent hypoperfusion and tissue hypoxia. Oxidative stress also occurs, in which endothelial dysfunction and capillary fluid and protein leaks occur. There is a loss of cellular barriers and tight junctions leading to worsening tissue edema [29].

Another area in which hemodynamic coherence may be affected is in hemorrhagic shock. Permissive hypotension and low volume fluid resuscitation are sometimes used in the initial stages of treatment and over time these can lead to insidious microcirculatory hypoperfusion. This may disrupt both coherence and cause a reperfusion injury. If this occurs, then monitoring and restoring the macrocirculation will not result in benefit to the microcirculation [30]. Loss of hemodynamic coherence has been associated with poor outcomes [24, 31].

As previously discussed, intravenous fluids are given to improve end-organ perfusion and oxygen delivery. Macrocirculatory parameters are used to deduce information about what is occurring at the microcirculatory level. However, as a lack of coherence may exist between the macro- and the microcirculation there is increasing evidence in favor of monitoring the effects of fluid at the microcirculatory level [22].

The microcirculation can be observed using a handheld camera at the patient's bedside. There are currently four generations of technology available. Through recording short video sequences of the microcirculation, information regarding fluid status can be ascertained. The images obtained can be scored and a number of measurements made. The microcirculation consensus meeting of 2007 described the following scoring systems: vessel density measurement including total vessel density and perfused vessel density and vessel perfusion assessment using proportion of perfused vessels and microcirculatory flow index (MFI). These parameters can be used to monitor the effects of fluid on the microcirculation [32]. Due to the limited availability of monitoring equipment and the need for offline analysis of images acquired, at present microcirculation measurement remains primarily a research tool [33].

The effect of intravenous fluid on the microcirculation varies depending on the underlying disease state. Shock can be broadly divided into four different classes: hypovolemic, distributive, cardiogenic and obstructive shock. Hypovolemic, cardiogenic and obstructive shock are associated with a low cardiac output. However, in sepsis, a form of distributive shock, cardiac output may be either low or high. In cardiogenic and obstructive shock there is increased afterload, with an expansion of the volume of the microcirculation. Hypovolemic and distributive shock are both characterized by impaired flow in the microcirculation [34]. However, the changes seen in the microcirculation in distributive shock are the most marked. Disruption occurs, with adjacent small vessels often exhibiting markedly different patterns of flow. Much of the research on the effects of fluids on the microcirculation has therefore focused on patients with sepsis [29]. The changes within the microcirculation that pre-date fluid administration must be considered to help predict the possible consequences of fluid administration.

Several mechanisms by which fluids exert their effects on the microcirculation have been described. The first, and arguably most important, is via increased flow. The effect of a fluid challenge on the macrocirculation, as previously described, increases filling within the system. In the volume-responsive patient this increases flow, which will increase microcirculatory perfusion by increasing pressure at the level of the capillaries.

Secondary effects relate to decreased viscosity secondary to hemodilution from fluid administration. The decreased viscosity will promote flow. In the hemoconcentrated patient this desirable feature will likely predominate; however, it may be that the hemodilution decreases oxygen carriage and cause shunting within the microcirculation [34].

Other adverse effects of fluid administration can be clearly demonstrated through direct vision of the microcirculation. Leakage of fluid extravascularly with increased tissue edema can be visualized and objectively monitored, as the vessel density will decrease. This results in increased diffusion distance from red blood cells to the tissues and decreased efficiency of oxygen delivery with subsequent hypoxia [29].

Measurement of flow within the microcirculation, at baseline, can be used to predict those that may benefit from a fluid challenge. Optimization of fluid status using macrocirculatory parameters does not always equate to improvement in clinical markers of hypoperfusion. Pranskunas et al. demonstrated that, in those with normal microcirculatory flow, no clinical benefit was gained by a fluid challenge, neither from the perspective of improvement in clinical markers of hypoperfusion nor an increase in MFI. In those with a low MFI, a significant improvement in MFI and clinical signs of hypoperfusion were seen following a fluid challenge [35]. Perioperatively, patients who develop postoperative complications have been shown to be more likely to have had microvascular flow abnormalities [36]. Those patients with a low MFI could not be identified by observing macrocirculatory parameters. Additionally, an increase in MFI did not correlate well with those who responded with an increase in stroke volume. The authors hypothesized this may be related to the fact that not all those who respond to a fluid challenge need the additional volume [35].

The effect of fluid administration on the microcirculation has been shown to vary dependent on the stage of 
the illness. In early sepsis, total vessel density, small vessel density and MFI all increased with fluid administration. The same effect was not seen in patients in the later stages of sepsis, defined as patients more than $48 \mathrm{~h}$ after diagnosis. These changes are not mirrored in the macrocirculation [37].

\section{Predicting Response to Fluids}

There are a number of different methods that can be used to try and predict which patients will be fluid responsive, prior to administering any fluid. Pulse pressure variation (PPV) and stroke volume variation (SVV) compare beat-to-beat variations, with a variation of more than $12 \%$ used as a marker of fluid responsiveness $[38,39]$. These methods are only validated for use in ventilated patients, with tidal volumes of more than $8 \mathrm{ml} / \mathrm{kg}$ and with no significant alteration in chest wall compliance. They can also only be used in patients in sinus rhythm [40].

Another predictor is vena cava collapsibility index. Variation in the diameter of the inferior vena cava (IVC) on transthoracic echocardiography is reasonably predictive of fluid responsiveness; however, measurement of the collapsibility of the superior vena cava on transesophageal echocardiography is more reliable. Measurement of the vena cava has the same limitations related to ventilation as PPV or SVV. It can, however, be used in patients with arrhythmias [40].

The end-expiratory occlusion test can also be used in patients receiving mechanical ventilation. Interruption of ventilation at end-expiration for at least $15 \mathrm{~s}$ causes an increase in preload. If cardiac output increases by more than $5 \%$ in response then this is predictive of fluid responsiveness [41].
Passive leg raise has gained increasing popularity as a method of assessing fluid responsiveness. It provides approximately $300 \mathrm{ml}$ of fluid as a challenge, increasing preload, from which fluid responsiveness can be determined. The technique can be reliably used in both ventilated and spontaneously ventilating patients. It provides a challenge of preload without the need to give intravenous fluids in patients who are then shown to be nonresponsive. However, it has its own limitations: for practical reasons it may not always be possible to perform and its reliability in the presence of intraabdominal hypertension has also been questioned [42].

As previously discussed it is important to try and predict the likely response to fluid administration prior to actually giving fluids. Figure 2 provides a simple flow chart of the possible decision pathway that a clinician may follow when considering fluid prescription for a patient in shock.

\section{Conclusion}

The decision to give intravenous fluid to a patient is a clinical one. The clinical assessment of each patient should include a prediction of whether it is likely that he/she will respond to additional volume and whether he/she requires and will benefit from it. Fluid administration is in general guided by the changes seen within the macrocirculation. Historically, this was presumed to represent the microcirculation; however, in illness, it has been shown that coherence may not exist. There are still many uncertainties regarding the effects of fluids on the microcirculation. The effects vary depending on the disease process and indeed the stage of the disease. At this stage, the effects of fluids on the microcirculation remain a focus of ongoing study and research.

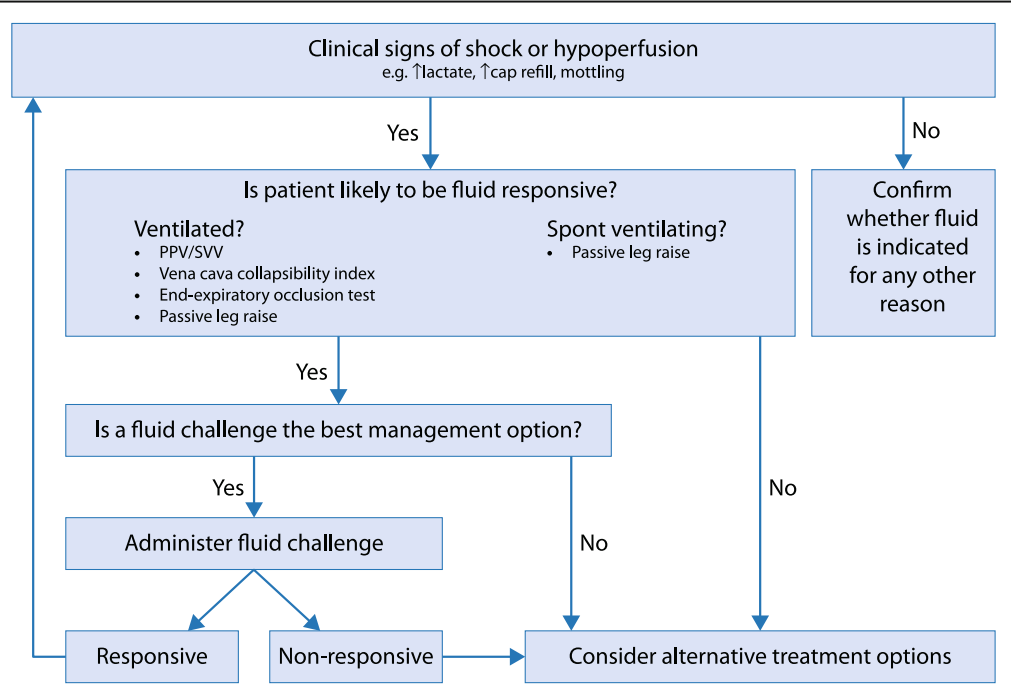

Fig. 2 Flow chart to demonstrate the possible decision-making process in fluid administration in shock. PPV: pulse pressure variation; SW: stroke volume variation 


\section{Acknowledgements}

VB and MC are also affiliated with St George's University of London.

\section{Funding}

Publication costs were funded by St George's University of London.

\section{Availability of data and materials}

Not applicable.

\section{Authors' contributions}

Initial draft was written by VB, AV, edited by MC. All authors had read and approved the final manuscript.

\section{Ethics approval and consent to participate}

Not applicable.

\section{Consent for publication}

Not applicable.

\section{Competing interests}

MC - consultancy for Edwards Lifesciences, LIDCO, Cheetah Medical,

\section{Publisher's Note}

Springer Nature remains neutral with regard to jurisdictional claims in published maps and institutional affiliations.

\section{Published online: 20 March 2018}

\section{References}

1. Padhi S, Bullock I, Li L, Stroud M, National Institute for Health and Care Excellence (NICE) Guideline Development Group. Intravenous fluid therapy for adults in hospital: summary of NICE guidance. BMJ. 2013;347:f7073.

2. Boyd JH, Forbes J, Nakada T, Walley KR, Russell JA. Fluid resuscitation in septic shock: a positive fluid balance and elevated central venous pressure are associated with increased mortality. Crit Care Med. 2011:39:259-65.

3. Vaara ST, Korhonen AM, Kaukonen K-M, et al. Fluid overload is associated with an increased risk for 90-day mortality in critically ill patients with rena replacement therapy: data from the prospective FINNAKI study. Crit Care. 2012;16:R197.

4. Liu V, Morehouse JW, Soule J, Whippy A, Escobar GJ. Fluid volume, lactate values, and mortality in sepsis patients with intermediate lactate values. Ann Am Thorac Soc. 2013:10:466-73.

5. Bellamy MC. Wet, dry or something else? Br J Anaesth. 2006;97:755-7.

6. Cecconi M, Hofer C, Teboul $\mathrm{L}$, et al. Fluid challenges in intensive care: the FENICE study: a global inception cohort study. Intensive Care Med. 2015;41:1529-37.

7. Gruartmoner G, Mesquida J, Ince C. Fluid therapy and the hypovolemic microcirculation. Curr Opin Crit Care. 2015;21:276-84.

8. Cecconi M, Parsons AK, Rhodes A. What is a fluid challenge? Curr Opin Crit Care. 2011;17:290-5

9. Charlton M, Sims M, Coats T, Thompson JP. The microcirculation and its measurement in sepsis. J Intensive Care Soc. 2016;18:221-7.

10. Patterson SW, Piper H, Starling EH. The regulation of the heart beat. J Physiol. 1914:48:465-513.

11. Patterson SW, Starling EH. On the mechanical factors which determine the output of the ventricles. J Physiol. 1914:48:357-79.

12. Aya HD, Rhodes A, Fletcher N, Grounds RM, Cecconi M. Transient stop-flow arm arterial-venous equilibrium pressure measurement: determination of precision of the technique. J Clin Monit Comput. 2016;30:55-61.

13. Aya HD, Ster IC, Fletcher N, Grounds RM, Rhodes A, Cecconi M. Pharmacodynamic analysis of a fluid challenge. Crit Care Med. 2015:44:880-91.

14. Cecconi M, Aya HD, Geisen $M$, et al. Changes in the mean systemic filling pressure during a fluid challenge in postsurgical intensive care patients. Intensive Care Med. 2013;39:1299-305.

15. Nixon JV, Murray RG, Leonard PD, Mitchell JH, Blomqvist CG. Effect of large variations in preload on left ventricular performance characteristics in normal subjects. Circulation. 1982:65:698-703.

16. Rivers E, Nguyen B, Havstad S, et al. Early goal directed therapy in the treatment of severe sepsis and septic shock. N Engl J Med. 2001;345:1368-77.

17. Dellinger RP, Levy MM, Carlet JM, et al. Surviving Sepsis Campaign: international guidelines for management of severe sepsis and septic shock: 2008. Intensive Care Med. 2008;34:17-60.
18. Bentzer P, Griesdale DE, Boyd J, MacLean K, Sirounis D, Ayas NT. Will this hemodynamically unstable patient respond to a bolus of intravenous fluids? JAMA. 2016;316:1298-309.

19. Marik PE, Cavallazzi R. Does the central venous pressure predict fluid responsiveness? An updated meta-analysis and a plea for some common sense. Crit Care Med. 2013:41:1774-81.

20. Marik PE, Baram M, Vahid B. Does central venous pressure predict fluid responsiveness? Chest. 2008;134:172-8.

21. Magdar S. Fluid status and fluid responsiveness. Curr Opin Crit Care. 2010; 16:289-96.

22. Ince $\mathrm{C}$. Hemodynamic coherence and the rationale for monitoring the microcirculation. Crit Care. 2015;19(Suppl 3):S8

23. Thiriet M. Macrocirculation. In: Lanzer P, editor. PanVascular Medicine. Berlin: Springer; 2014. p. 1-54.

24. Arnemann $\mathrm{P}$, Seidel L, Ertmer C. Haemodynamic coherence - the relevance of fluid therapy. Best Pract Res Clin Anaesthesiol. 2016;30:419-27.

25. Van Iterson M, Bezemer R, Heger M, Siegemund M, Ince C. Microcirculation follows macrocirculation in heart and gut in the acute phase of hemorrhagic shock and isovolemic autologous whole blood resuscitation in pigs. Transfusion. 2012:52:1552-9.

26. Siegemund M, Van Bommel J, Sinaasappel M, et al. The NO donor SIN-1 improves intestinal-arterial Pco2 gap in experimental endotoxemia: an animal study. Acta Anaesthesiol Scand. 2007;51:693-700.

27. Almac $E$, Siegemund $M$, Demirci $C$, Ince C. Microcirculatory recruitment maneuvers correct tissue $\mathrm{CO} 2$ abnormalities in sepsis. Minerva Anestesiol. 2006;72:509-19.

28. Lush CW, Kvietys PR. Microvascular dysfunction in sepsis. Microcirculation. 2000;7:83-101.

29. Elbers PWG, Ince C. Mechanisms of critical illness - classifying microcirculatory flow abnormalities in distributive shock. Crit Care. 2006;10:221.

30. Libert N, Harrois A, Duranteau J. Haemodynamic coherence in haemorrhagic shock. Best Pract Res Clin Anaesthesiol. 2016;30:429-35.

31. Vellinga NA, Ince C, Boerma EC. Elevated central venous pressure is associated with impairment of microcirculatory blood flow in sepsis: a hypothesis generating post hoc analysis. BMC Anesthesiol. 2013:13:17.

32. De Backer D, Hollenberg S, Boerma C, et al. How to evaluate the microcirculation: report of a round table conference. Crit Care. 2007:11:R101.

33. Tafner PFDA, Chen FK, Filho RR, Corrêa TD, Chaves RCF, Neto SA. Recent advances in bedside microcirculation assessment in critically ill patients. Rev Bras Ter Intensiva. 2017:29(2):238-47.

34. Boldt J, Ince C. The impact of fluid therapy on microcirculation and tissue oxygenation in hypovolemic patients: a review. Intensive Care Med. 2010;36: 1299-308.

35. Pranskunas A, Koopmans M, Koetsier PM, Pilvinis V, Boerma EC. Microcirculatory blood flow as a tool to select ICU patients eligible for fluid therapy. Intensive Care Med. 2013;39:612-9.

36. Jhanji S, Lee C, Watson D, Hinds C, Pearse RM. Microvascular flow and tissue oxygenation after major abdominal surgery: Association with post-operative complications. Intensive Care Med. 2009;35:671-7.

37. Ospina-Tascon $G$, Neves AP, Occhipinti $G$, et al. Effects of fluids on microvascular perfusion in patients with severe sepsis. Intensive Care Med. 2010;36:949-55.

38. De Backer D, Heenen S, Piagnerelli M, Koch M, Vincent JL. Pulse pressure variations to predict fluid responsiveness: influence of tidal volume. Intensive Care Med. 2005:31:517-23.

39. Marik PE, Cavallazzi R, Vasu T, Hirani A. Dynamic changes in arterial waveform derived variables and fluid responsiveness in mechanically ventilated patients: a systematic review of the literature. Crit Care Med. 2009;37:2642-7

40. Monnet $X$, Marik PE, Teboul JL. Prediction of fluid responsiveness: an update. Ann Intensive Care. 2016:6:111.

41. Monnet X, Osman D, Ridel C, Lamia B, Richard C, Teboul JL. Predicting volume responsiveness by using the end-expiratory occlusion in mechanically ventilated intensive care unit patients. Crit Care Med. 2009:37.951-6.

42. Monnet $X$, Marik P, Teboul JL. Passive leg raising for predicting fluid responsiveness: a systematic review and meta-analysis. Intensive Care Med. 2016;42:1935-47. 